\title{
Molecular dynamics simulations of the interaction of carbon nanotube and a carbon disulfide solvent
}

\author{
Kholmirzo Kholmurodov ${ }^{1,2 *}$, Guzel Aru ${ }^{1}$, Kenji Yasuoka ${ }^{3}$ \\ ${ }^{1}$ Laboratory of Radiation Biology, Joint Institute for Nuclear Research, Dubna, Russia; *Corresponding Author: mirzo@jinr.ru \\ ${ }^{2}$ International University "Dubna”, Dubna, Russia \\ ${ }^{3}$ Department of Mechanical Engineering, Keio University, Yokohama, Japan
}

Received 4 February 2010; revised 18 March 2010; accepted 24 March 2010.

\begin{abstract}
An analysis of the molecular dynamics (MD) of the interaction between a carbon nanotube (CNT) and a carbon disulfide active solvent $\left(\mathrm{CS}_{2}\right)$ has been carried out. The aim of the present work is to estimate the dynamical and structural behavior of the CNT-CS ${ }_{2}$ system at different relative atomic concentrations and under temperature changes. The structural radial distribution functions and the dynamical configurations have been built for a CNT interacting with a $\mathrm{CS}_{2}$ solvent. A nontrivial observation for the CNT-CS $_{2}$ system is that the solvent carbon disulfide atoms make up a patterned (layered) formation around the carbon nanotube.
\end{abstract}

Keywords: Molecular Dynamics; Carbon Nanotube; Carbon Disulfide Solvent

\section{INTRODUCTION}

Among the organic materials, carbon Nanotubes (CNTs) are unique for their electrical and chemical properties. They are very interesting in terms of material research and electronic applications. Depending on their chemical structure, carbon nanotubes (CNTs) can be used as an alternative to organic or inorganic semiconductors as well as conductors. The chemical bonding of nanotubes is composed entirely of $\mathrm{sp}^{2}$ bonds, similar to those of graphite. This bonding structure, which is stronger than the $\mathrm{sp}^{3}$ bonds found in diamonds, provides the molecules with their unique strength. Nanotubes naturally align themselves into "ropes" held together by van der Waals forces. The nature of the bonding of a nanotube is described by quantum chemistry-specifically, orbital hybridization. Solvents in which the CNTs can be solubilized include chlorobenzene, chloroform, methylene chloride, carbon disulfide, benzene, etc. The solubilities of the carbon nanotubes in these solvents range from about 0.01 to $5.0 \mathrm{mg} / \mathrm{ml}[1-4]$.

The aim of the present paper is to simulate the dynamical and structural properties of a CNT interacting with a carbon disulfide $\left(\mathrm{CS}_{2}\right)$ solvent taking into account the Van der Waals forces only. For the CNT-CS 2 system, we simulate different relative CNT solvent concentrations and temperature scales. In the description of the physical properties of the CNT, we employ the Tersoff potential [5]. It is a special case of a density-dependent potential, which reproduces the properties of the covalent bonding in systems containing carbon, silicon, germanium, etc, and alloys of these elements. A special feature of the potential is that it allows bond breaking and associated changes in bond hybridization. The energy is modelled as a sum of pair-like interactions where, however, the coefficient of the attractive term in the pairlike potential (which plays the role of the bond order) depends on the local environment giving a many-body potential.

The Tersoff potential has 11 atomic and 2 bi-atomic parameters (see, formulas 1-9):

$$
U_{i j}=f_{C}\left(r_{i j}\right)\left[f_{R}\left(r_{i j}\right)-\gamma_{i j} f_{A}\left(r_{i j}\right)\right],
$$

where the potential parameters have the following forms:

$$
\begin{gathered}
f_{R}\left(r_{i j}\right)=A_{i j} \exp \left(-a_{i j} r_{i j}\right), \\
f_{A}\left(r_{i j}\right)=B_{i j} \exp \left(-b_{i j} r_{i j}\right), \\
f_{C}\left(r_{i j}\right)=\frac{1}{2}+\frac{1}{2} \cos \left[\pi\left(r_{i j}-R_{i j}\right) /\left(r_{i j}-R_{i j}\right)\right], R_{i j}<r_{i j}<S_{i j},
\end{gathered}
$$

In (1) $f_{R}\left(r_{i j}\right)$ and $f_{A}\left(r_{i j}\right)$ mean repulsive and attractive, $f_{C}\left(r_{i j}\right)$-potential cutoff functions $\left(f_{C}\left(r_{i j}\right)=\right.$ $f_{C}\left(r_{i j}\right)=1$ for $r_{i j}<R_{i j}$ and $f_{C}\left(r_{i j}\right)=0$ for $\left.r_{i j}>S_{i j}\right)$.

It is worth noting that the main feasure of the Tersoff potential is that the coefficients in (1) reflect many-body 
nature of the interactions. The basic means of the coefficients is that the strenth of each bond depends on the local environment and is lowered when the number of neighboors is relatively high. This dependence can accentuate or diminish the attractive force relative to the repulsive force, according to the environment, such that:

$$
\begin{gathered}
\gamma_{i j}=\chi_{i j}\left(1+\beta_{i}^{\eta_{i}} L_{i j}^{\eta_{i}}\right)^{-1 / 2 \eta_{i}}, \\
L_{i j}=\sum_{k \neq i, j} f_{C}\left(r_{i k}\right) \omega_{i k} g\left(\theta_{i j k}\right), \\
g\left(\theta_{i j k}\right)=1+c_{i}^{2} / d_{i}^{2}-c_{i}^{2} /\left[d_{i}^{2}+\left(h_{i}-\cos \theta_{i j k}\right)^{2}\right], \\
a_{i j}=\left(a_{i}+a_{j}\right) / 2, \quad b_{i j}=\left(b_{i}+b_{j}\right) / 2, \\
A_{i j}=\left(A_{i} A_{j}\right)^{1 / 2}, B_{i j}=\left(B_{i} B_{j}\right)^{1 / 2}, A_{i j}=\left(A_{i} A_{j}\right)^{1 / 2}, \\
R_{i j}=\left(R_{i} R_{j}\right)^{1 / 2}, S_{i j}=\left(S_{i} S_{j}\right)^{1 / 2} .
\end{gathered}
$$

We have accepted the following values: $\chi_{i i}=1$, $\chi_{i j}=\chi_{j i}, \omega_{i i}=1, \omega_{i j}=\omega_{j i}$.

The carbon disulfide $\left(\mathrm{CS}_{2}\right)$ solvent has a comparatively high solubility $(\sim 7.9 \mathrm{mg} / \mathrm{ml})$. Several earlier papers considered interaction between the $\mathrm{CS}_{2}$ solvent and a fullerene $\left(\mathrm{C}_{60}\right)$ solution [6-12]. The $\mathrm{C}_{60}-\mathrm{CS}_{2}$ system belongs to a class of solutions where a peak in the temperature dependence of solubility is observed $\left(\mathrm{T}_{\max } 280 \mathrm{~K}\right)$. The structural features of the fullerene in a solution, as well as the fullerene-solvent $\left(\mathrm{C}_{60}-\mathrm{CS}_{2}\right)$ interaction mechanism have been investigated in detail in [6-12] by different methods (small-angle neutron scattering (SANS) and others). As was noted in [12], the characteristic size of the $\mathrm{CS}_{2}$ molecule $(\sim 0.3 \mathrm{~nm})$ is comparable to that of the $\mathrm{C}_{60}$ fullerene $(\sim 1 \mathrm{~nm})$; so, any interface organization of the $\mathrm{CS}_{2}$ molecules different from that in bulk must result in a significant difference between the interface and bulk molecular density of the solvent, and, hence, affect the visible size of the fullerene.

\section{MATERIALS AND METHODS}

We performed the molecular dynamics (MD) simulation of several CNT-CS $\mathrm{CS}_{2}$ model systems. The MD simulation was based on the DL_POLY general-purpose code [13-15]. The MD cell is orthorhombic and square in the $\mathrm{XY}$ plane $(30.7 \times 30.7 \times 41.7)$. The integration algorithm is an NPT Berendsen 'ensemble'.
The CNT (carbon nanotube) consists of 800 carbon atoms in a nanotube of 41.7 angstrom in length (see Figure 1). For the CNT, we used the Tersoff potential parameters of the DL_POLY software database [13-14]:

$$
A=1393.6, \quad a=3.4879, \quad B=346.74, \quad b=2.2119 \text {, }
$$
$R=1.8, S=2.1$,

$\beta=1.5724 \times 10^{-7}, \eta=0.72751, c=38049, d=$ 4.3484, $h=-0.57058$.

The $\mathrm{CS}_{2}$ molecules were treated as rigid with the bond length of 1.55 angstrom between carbon and sulfide atoms (Figure 2). Throughout the computation, only the Lennard-Jones (LJ) interactions were taken into account. The number of the $\mathrm{CS}_{2}$ solvent molecules was varied, so we simulated CNT-solvent model systems of different relative atomic concentrations: $\mathrm{x}=0.2,0.4, \ldots, 1.0$ (Figure 3). The LJ potential was also used for the CNTsolvent interactions; the potential and parameteres are shown in Table 1, where C denotes the CNT carbon

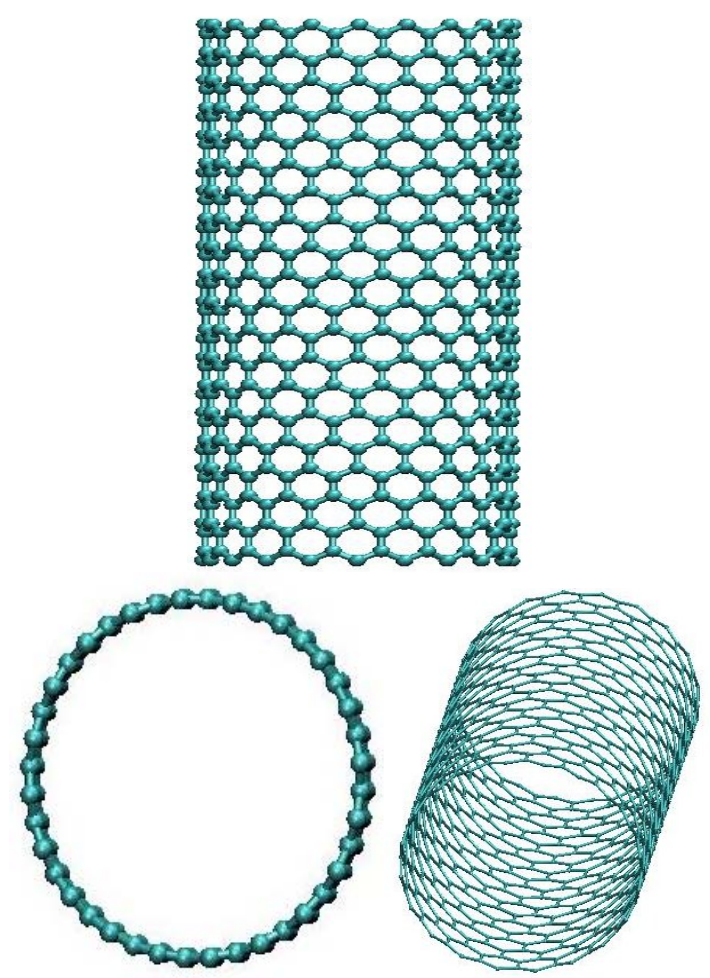

Figure 1. Structural presentations of the carbon nanotube (CNT) (top and bottom).

Table 1. Potential parameters of the CNT-CS 2 model.

\begin{tabular}{cccccc}
\hline Atomic pair & Potential & Functional form & Parameters & $\boldsymbol{\varepsilon}, \mathbf{e V}$ & $\boldsymbol{\sigma}, \boldsymbol{\AA}$ \\
\hline $\mathrm{C}_{-} \mathrm{C}_{S}$ & $\mathrm{Lj}$ & $U(r)=4 \varepsilon\left[\left(\frac{\sigma}{r}\right)^{12}-\left(\frac{\sigma}{r}\right)^{6}\right]$ & $\boldsymbol{\varepsilon}, \boldsymbol{\sigma}$ & 0.0044 & 3.35 \\
$\mathrm{C}-\mathrm{S}$ & $\mathrm{Lj}$ & $\ldots$ & $\boldsymbol{\varepsilon}, \boldsymbol{\sigma}$ & 0.0082 & 3.44 \\
$\mathrm{~S}-\mathrm{S}$ & $\mathrm{Lj}$ & $\ldots$ & $\boldsymbol{\varepsilon}, \boldsymbol{\sigma}$ & 0.0153 & 3.52 \\
\hline
\end{tabular}




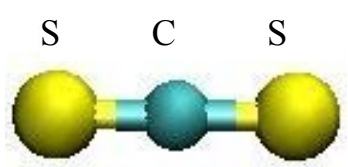

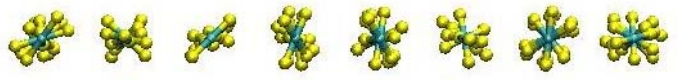

\%

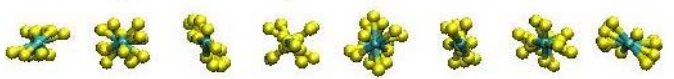

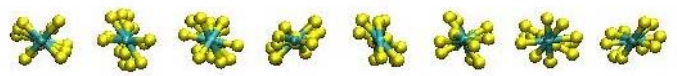

篅

I

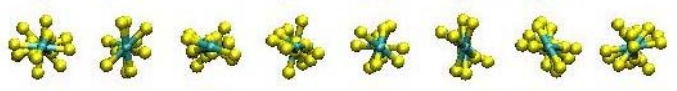

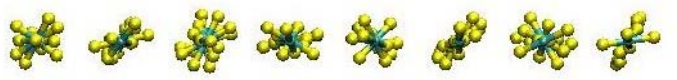

Figure 2. Structural presentations of the $\mathrm{CS}_{2}$ solvent (top and bottom).

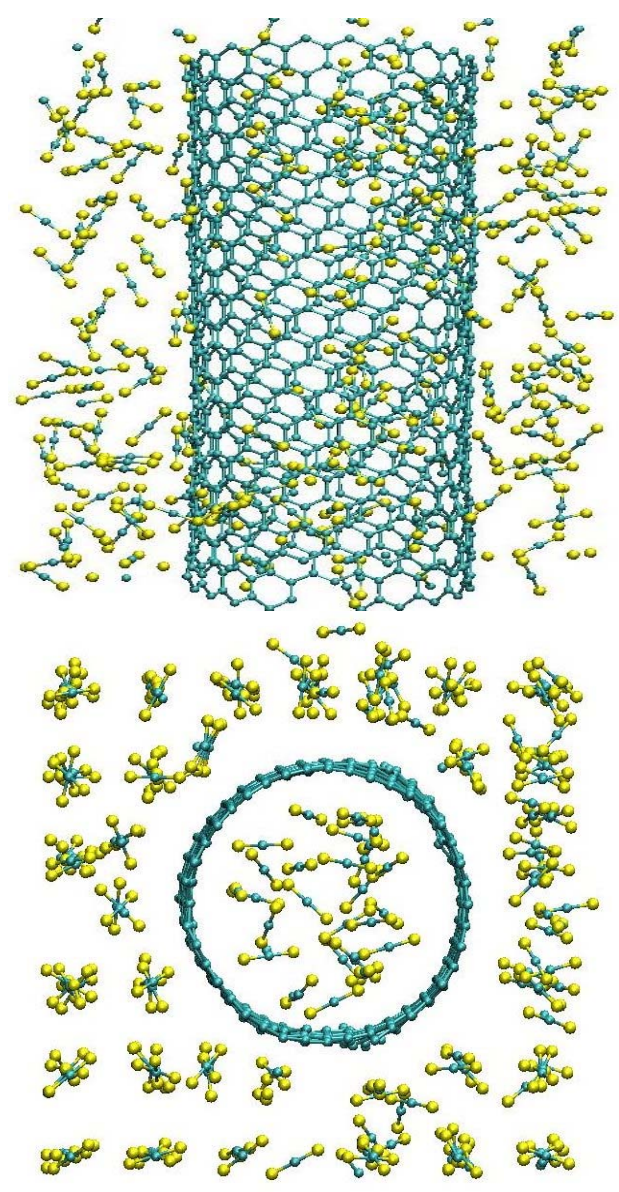

Figure 3. Structural presentations of the CNT$\mathrm{CS}_{2}$ system (top and bottom). atoms and $\mathrm{C}_{\mathrm{s}}$ - the carbon atoms of the $\mathrm{CS}_{2}$ solvent.

\section{RESULTS AND DISCUSSIONS}

The dynamics of the interaction between the CNT (carbon nanotube) and the $\mathrm{CS}_{2}$ solvent has been studied at different ratios of the CNT-solvent atomic concentrations:

$$
x=\frac{N_{S}}{N_{C N T}}
$$

where $N_{s}$ is the number of the solvent atoms and $N_{C N T}$ is the number of the CNT atoms. We have simulated five $\mathrm{CNT}^{-\mathrm{CS}_{2}}$ systems with $\mathrm{x}=0.2,0.4,0.6,0.8$ and 1 . We call the systems with $\mathrm{x}=0.2$ and $\mathrm{x}=1$ low- and high-density systems, respectively. The CNT consists of 800 carbon (C) atoms. Further, we denote the carbon atoms of the $\mathrm{CS}_{2}$ solvent as $\mathrm{C}_{\mathrm{s}}$.

\subsection{Structural RDFs for the CNT-Solvent Atomic Pairs}

In Figure 4, we present the behavior of the radial
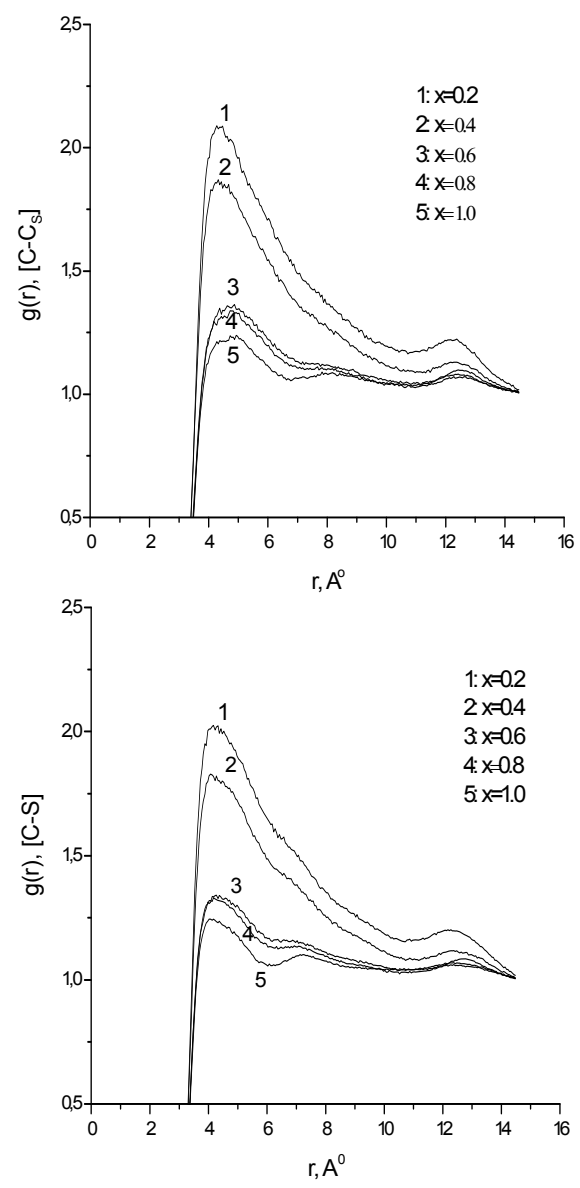

Figure 4. Structural RDFs for the atomic pairs $\mathrm{C}-\mathrm{Cs}$ and $\mathrm{C}-\mathrm{S}$ at different ratios of the CNTsolvent atomic concentration $\mathrm{x}$. 
distribution function (RDF) for the CNT-solvent atomic pairs $\mathrm{C}-\mathrm{C}_{\mathrm{s}}$ and $\mathrm{C}-\mathrm{S}$ at different values of $\mathrm{x}$. It can be seen that the RDF of the CNT-CS 2 system at $\mathrm{x}>0.4$ obeys a similar law. For all values of $x$, we have clearly observed two peaks in the RDF graphs. However, for $\mathrm{x}>0.4$, Figure 4 shows an additional small RDF peak between the first two ones. This behavior of the RDF points to a structural rearrangement of the $\mathrm{CNT}-\mathrm{CS}_{2}$ system, which is going from the low density phase $(\mathrm{x}=0.2)$ into the high density phase $(x=1)$. The additional (third) peak in the RDF curve has also been observed on the temperature dependence (part III below).

\subsection{Structural RDFs for the Solvent-Solvent Atomic Pairs}

In Figures 5 and 6, the radial distribution functions (RDFs) are presented for the solvent-solvent atomic pairs $\left(\mathrm{C}_{\mathrm{s}}-\mathrm{C}_{\mathrm{s}}, \mathrm{C}_{\mathrm{s}}-\mathrm{S}\right.$, and $\left.\mathrm{S}-\mathrm{S}\right)$ at different values of $\mathrm{x}$. It is seen that the RDFs of the solvent atoms differ from each other by their first peaks only; the secondary peaks for all solvent-solvent atomic pairs $\left(\mathrm{CS}_{2}-\mathrm{CS}_{2}\right)$ are similar. The RDF for the S-S atoms has a comparably large first
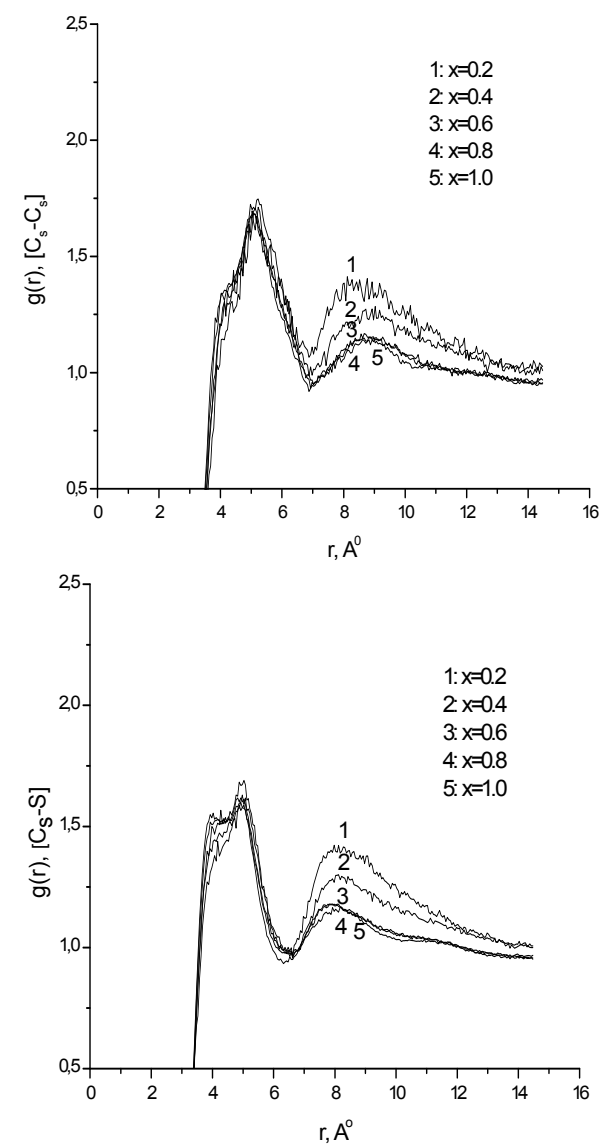

Figure 5. Structural RDF for the atomic pairs Cs-Cs and Cs-S at different ratios of the CNTsolvent atomic concentration $\mathrm{x}$.

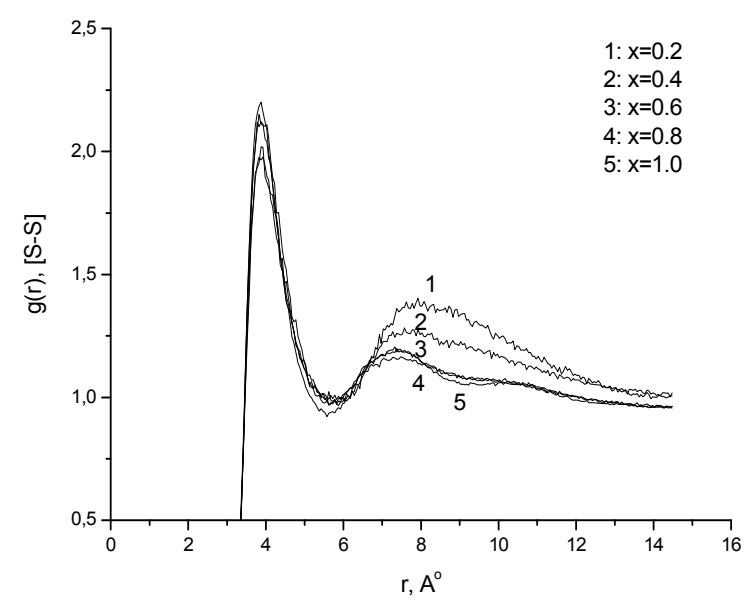

Figure 6. Structural RDF for the atomic pair S-S at dif ferent ratios of the $\mathrm{CNT}-$ solvent atomic concentration $\mathrm{x}$.

peak (Figure 6). This indicates that in the solvent media, the atomic pair $\mathrm{S}-\mathrm{S}$ has a relatively high ordering in comparison with the $\mathrm{C}_{\mathrm{s}}-\mathrm{C}_{\mathrm{s}}$ one. The RDF first peak for the $\mathrm{C}_{\mathrm{s}}-\mathrm{S}$ atomic pair (Figure 5, below) is low as compared with S-S ones. We attribute such behavior to an influence of CNT's carbon (C) atoms on the CNTsolvent interaction process and ordering.

\subsection{MD-Simulated Structural CNT-CS Configurations}

We have compared the MD structural configurations of the CNT-CS $\mathrm{CS}_{2}$ system for the low-density $(\mathrm{x}=0.2)$ and high-density $(x=1)$ phases. In Figure 7, MD-simulated snapshots are presented for $x=0.2$. Figure 7 shows the side and top views (left and righr, respectively) of the $\mathrm{CNT}_{-} \mathrm{CS}_{2}$ system; the snapshots correspond to the moments of $\mathrm{t}=0$ (top), $10 \mathrm{ps}$ (middle), and $80 \mathrm{ps}$ (bottom). It is seen that starting from arbitrarily distributed positions at the initial $(\mathrm{t}=0)$ state, the solvent $\left(\mathrm{CS}_{2}\right)$ atoms become more structured in the later states of the dynamics around and inside the CNT.

The $\mathrm{CS}_{2}$ structuring behavior around the CNT has also been observed during temperature variation in the $\mathrm{CNT}_{-} \mathrm{CS}_{2}$ system. In Figure 8, the RDF curves are displayed for the low density phase $(\mathrm{x}=0.2)$ depending on temperature: $\mathrm{T}=200 \mathrm{~K}(1), \mathrm{T}=250 \mathrm{~K}(2)$, and $\mathrm{T}=$ $300 \mathrm{~K}$ (3). Figure 8 shows the RDF results for CNT-solvent atomic pairs $\mathrm{C}_{-} \mathrm{C}_{\mathrm{s}}$ (left) and C-S (right). The RDFs in Figure 8 show some RDF changes (only for the first peaks).

Figure 9 shows RDF curves for the solvent-solvent atomic pairs $\mathrm{C}_{\mathrm{s}}-\mathrm{C}_{\mathrm{s}}$ (left) and $\mathrm{C}_{\mathrm{s}}-\mathrm{S}$ (right). A visible change in the RDF graph is seen for the atomic pair $\mathrm{C}_{\mathrm{s}}-\mathrm{S}$. During the temperature variation, we observe changes for $\mathrm{C}_{\mathrm{s}}-\mathrm{S}$ both in the first and secondary peaks.

$A$ is seen in the RDF graph in Figure 10, the atomic 
(a) Low-density phase $(\mathrm{x}=\mathbf{0 . 2})$
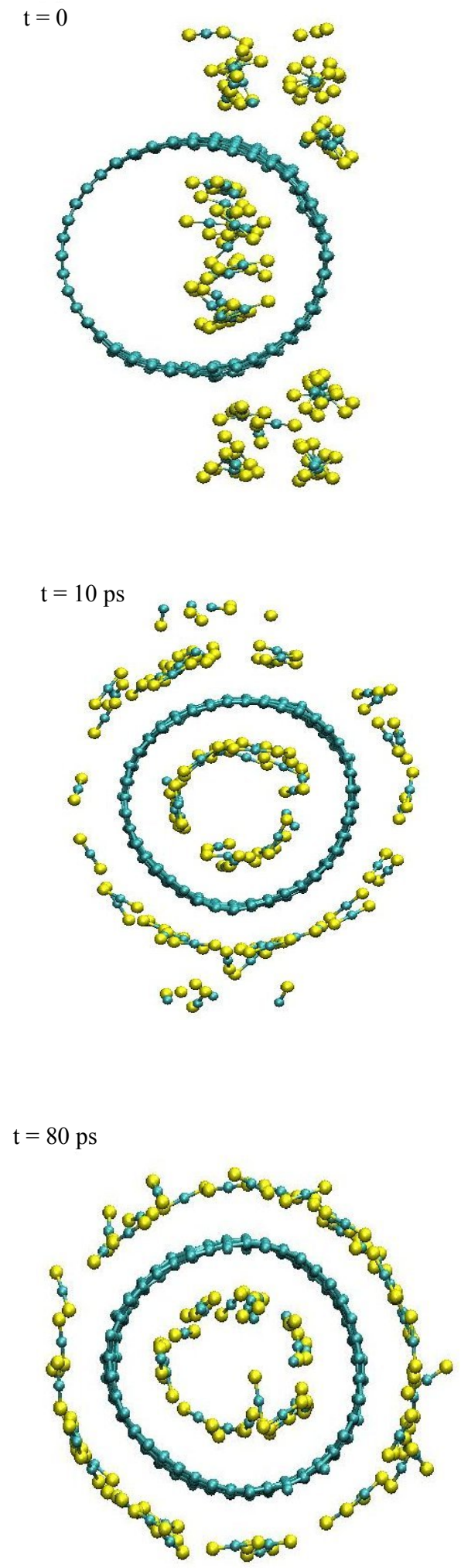
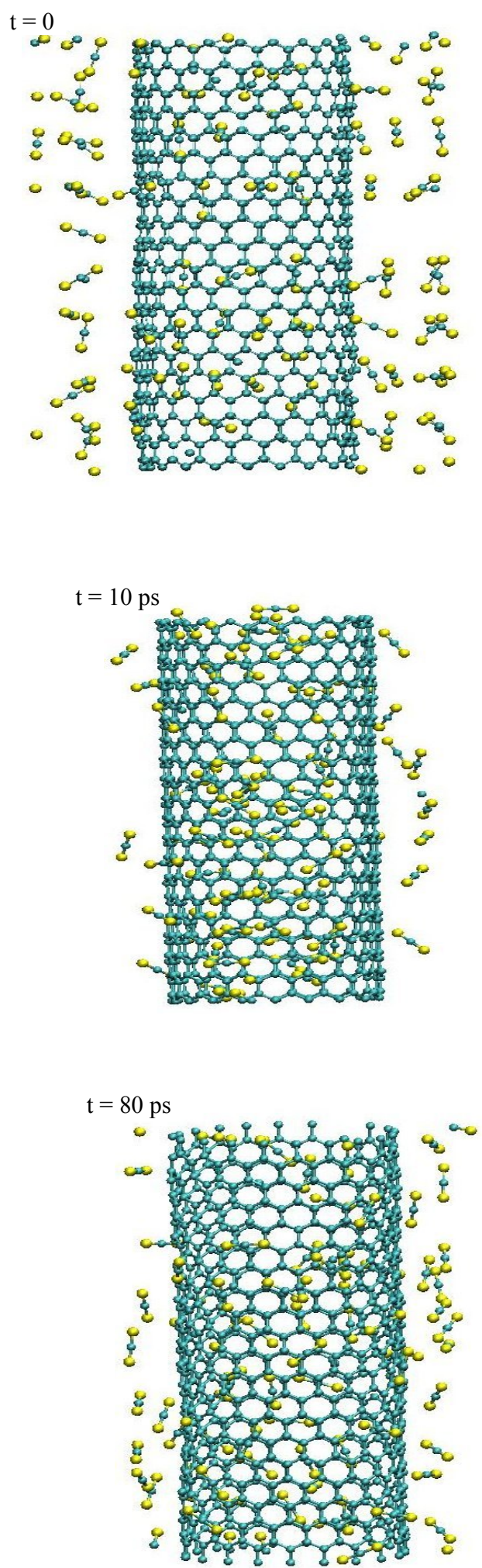

Figure 7. Snapshots of the CNT-solvent configurations at a low density (relative atomic concentration $\mathrm{x}=0.2$ ) 

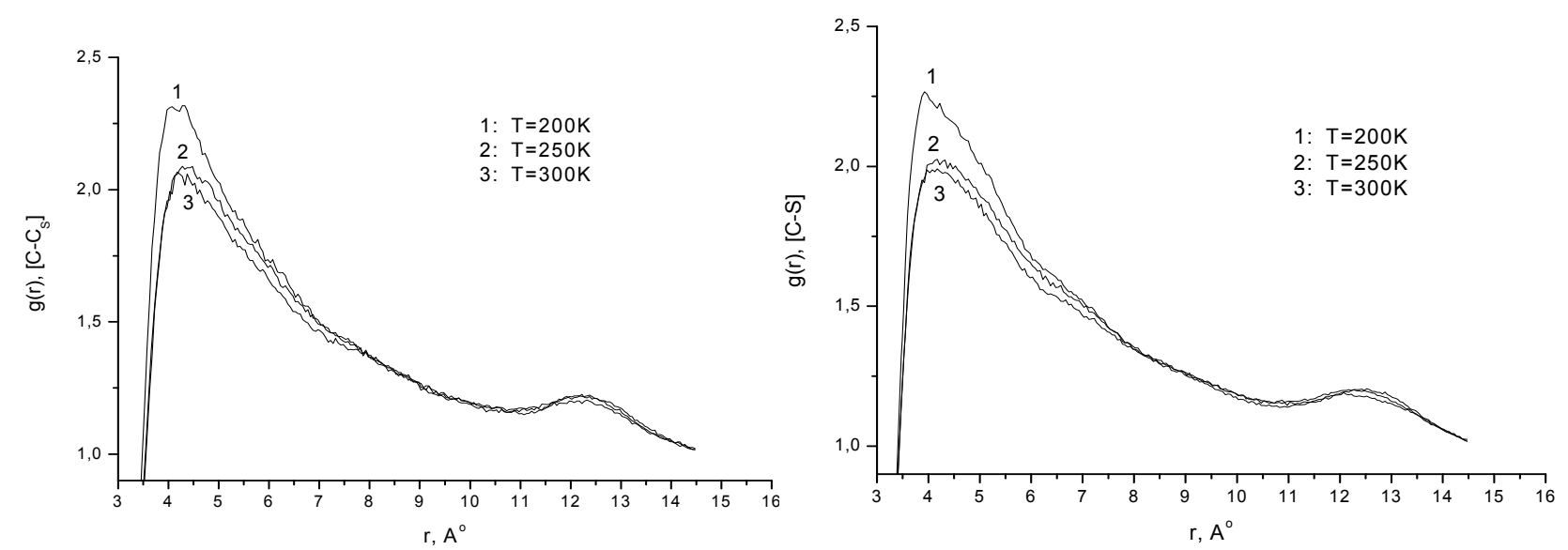

Figure 8. Structural RDFs for the atomic pairs C-Cs and C-S at $\mathrm{x}=0.2$ with temperature.
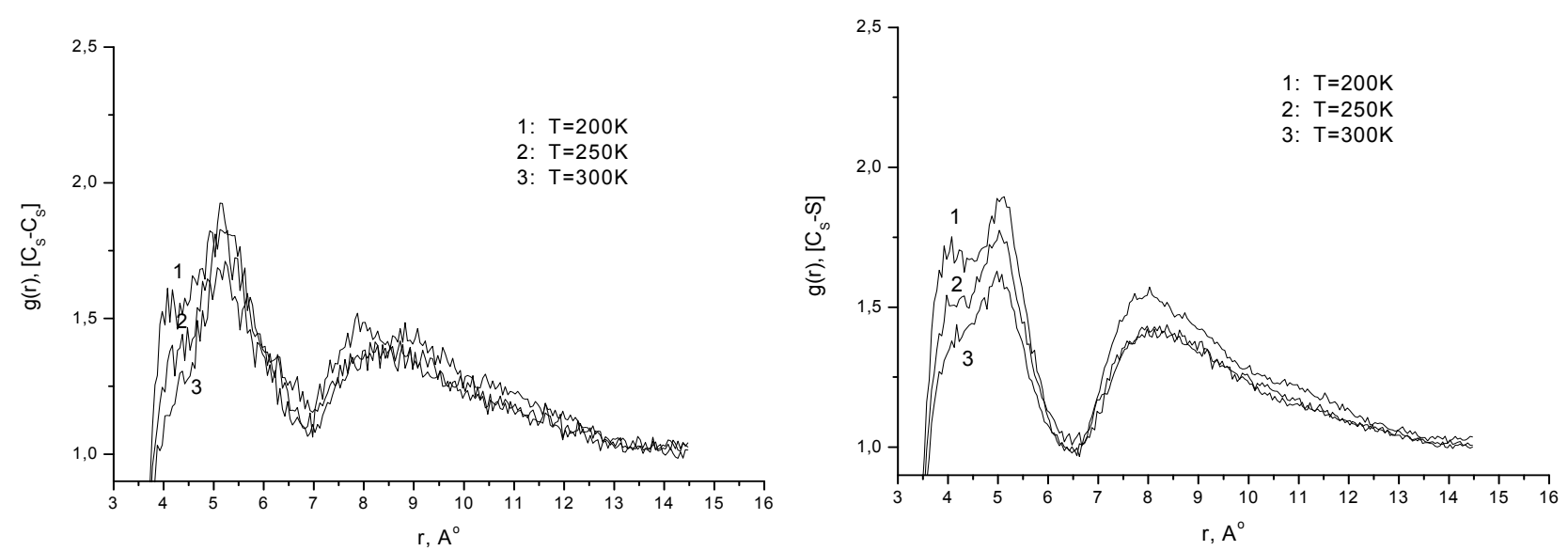

Figure 9. Structural RDF for the atomic pairs Cs-Cs and Cs-S at $x=0.2$ with temperature.

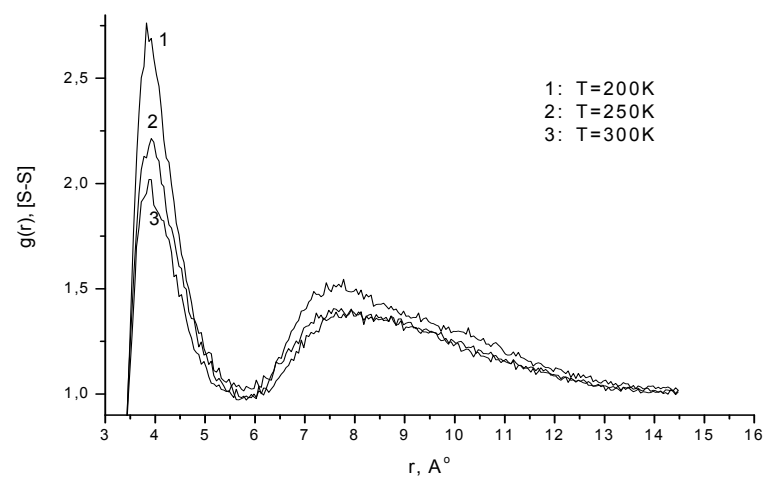

Figure 10. The structural RDF for the atomic pair S-S at $x$ $=0.2$ with temperature.

pair S-S has a relatively high ordering in the solvent media. Also, the RDF for the S-S pair shows a strong temperature dependence. We see that the amplitude of the first peak decreases twice as the temperature increases from $\mathrm{T}=200 \mathrm{~K}$ to $300 \mathrm{~K}$.

In Figure 11, the MD snapshots are presented for the high density phase $(x=1)$.
Comparing these results with those of the low density phase $(x=0.2$, Figure 7$)$, we observe a similar structural formation of the $\mathrm{CS}_{2}$ solvent atoms around the CNT. However, the RDFs of the high-density phase are strongly specific against the low-density phase RDFs. A comparison of Figures 12-14 with Figures 8-10 is straightforward.

\subsection{Patterned Structure Formation in the CNT-CS $_{2}$ System}

One of the nontrivial observations for the CNT-CS system is that the solvent carbon disulfide atoms make up a patterned (layered) formation around the carbon nanotube. In Figure 15, we present a CNT-CS ${ }_{2}$ resultant structure where the atomic distributions are compared in three regions (marked by the circles 1,2 , and 3 ). It is seen that in regions 1 and 2 , the solvent $\mathrm{CS}_{2}$ atoms have to be configured similarly to CNT's shape. The solvent atoms inside and outside the CNT are regularly distributed within the spheres of the same radii off the CNT. In contrast, for region 3 we observe an 
(b) High density phase $(x=1)$
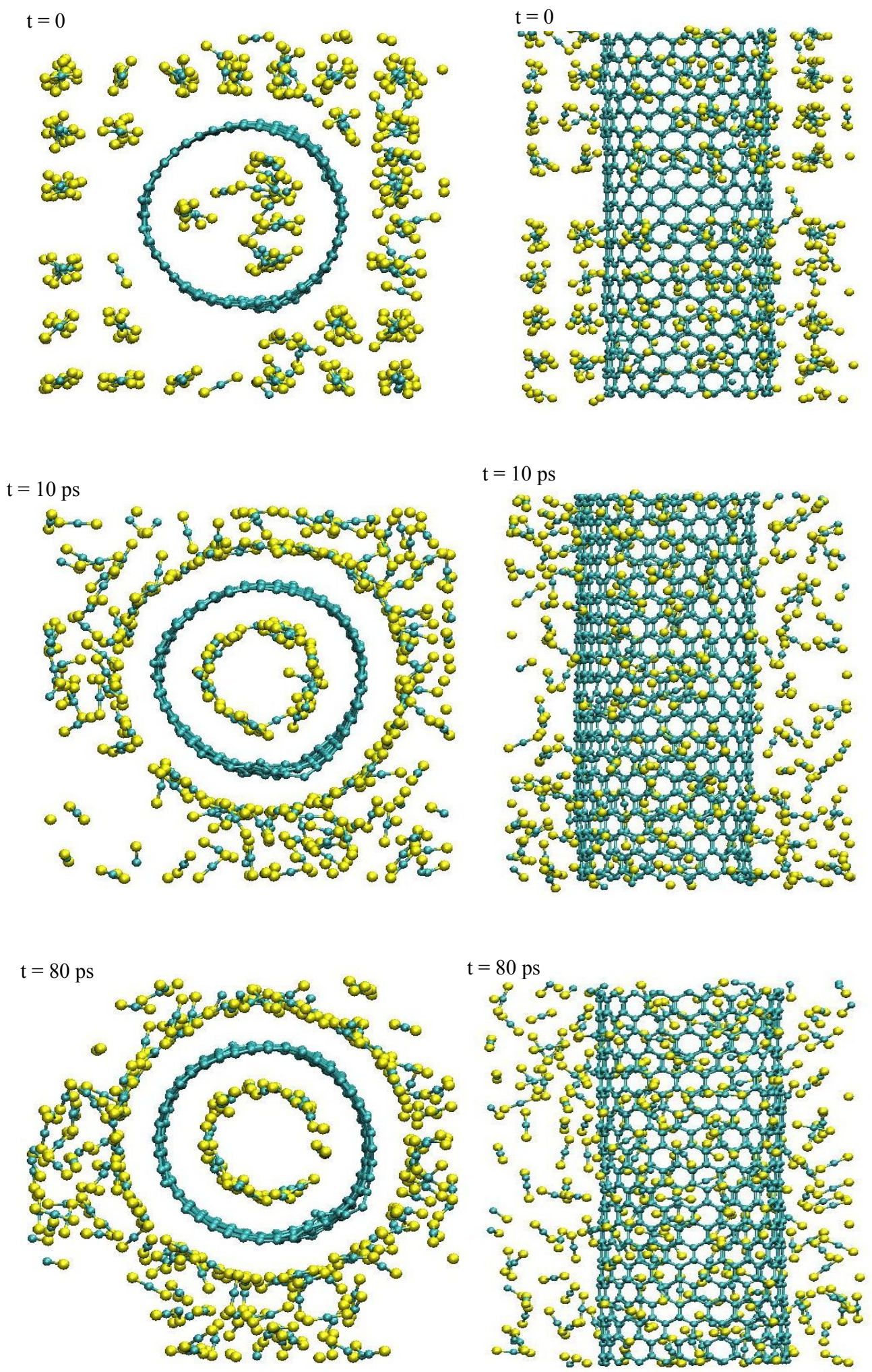

Figure 11. Snapshots of the CNT - solvent configurations at a high density (relative atomic concentration $\mathrm{x}=1$ ). 

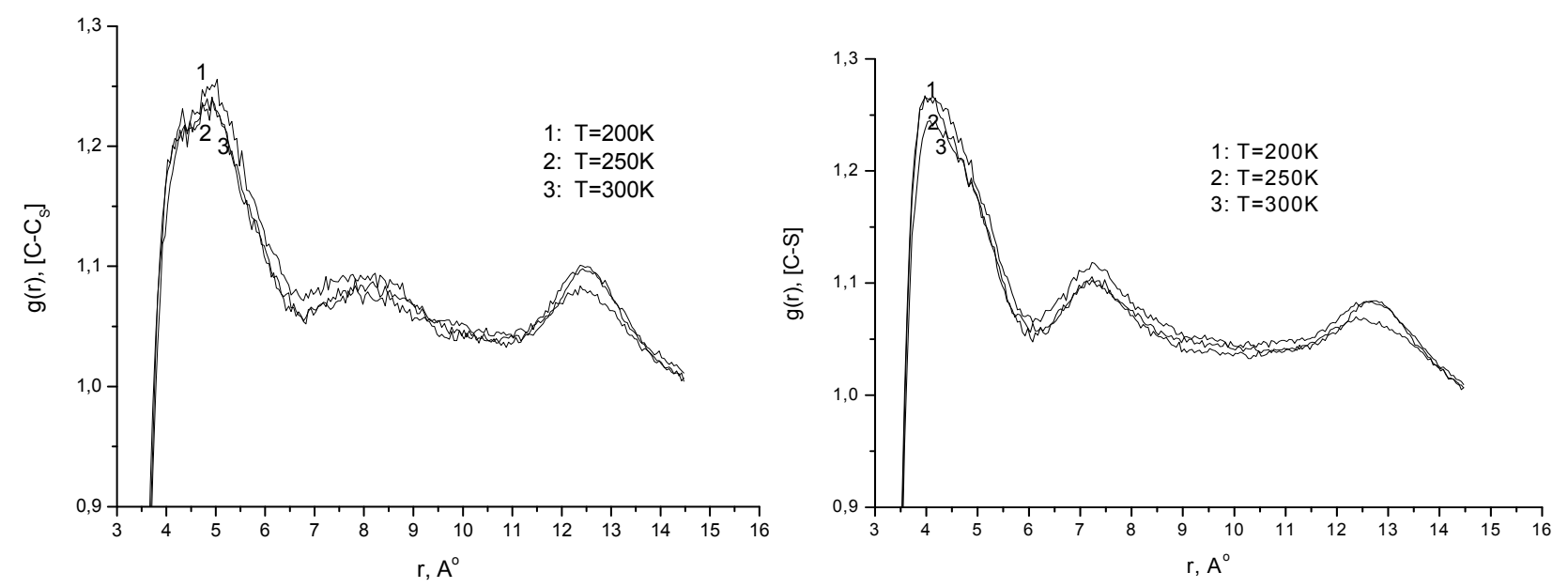

Figure 12. Structural RDFs for the atomic pairs $C-C s$ and $C-S$ at $x=1$ with temperature.
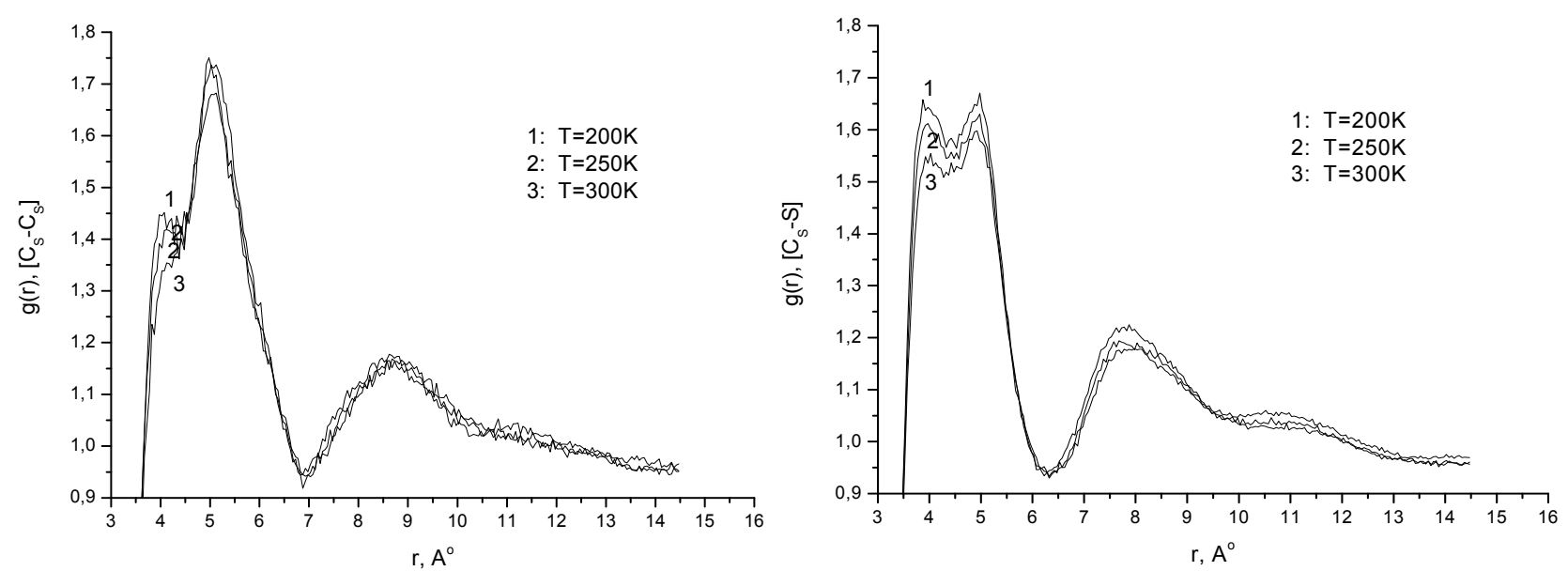

Figure 13. Structural RDFs for the atomic pairs Cs-Cs and Cs-S at $x=1$ with temperature.

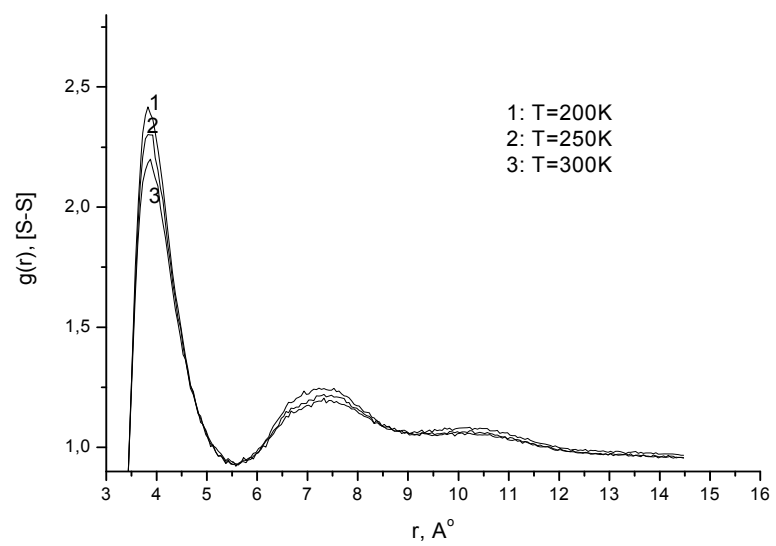

Figure 14. Structural RDF for the atomic pair $S-S$ at $x=1$ with temperature.

irregular structure of the same atoms that results from solvent-solvent ineraction. It should be noted that Figure 15 shows an important example of a graphene-

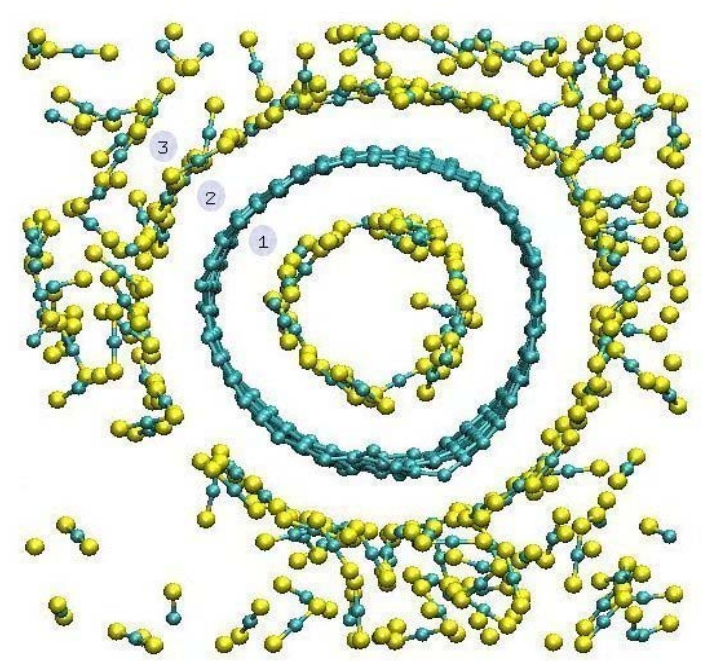

Figure 15. The CNT-CS2 atomic distributions for three regions shown as circles 1,2 , and 3 . 
like (patterned, layered) behavior. Such formations are of great importance for the applications and technological uses of the CNT-CS 2 systems [1-12].

\section{ACKNOWLEDGEMENTS}

This work has been fulfilled under joint collaboration agreements Daresbury Laboratory, UK - Keio University, Japan - JINR, Russia. This work was supported in part by Grant in Aid for the Global Center of Excellence Program for "Center for Education and Research of Symbiotic, Safe and Secure System Design" from the Ministry of Education, Culture, Sport, and Technology in Japan. We thanks Prof. William Smith (Daresbury Laboratory, UK) for the software support. We would like to thank Prof. Mikhail V. Altaisky (the Joint Institute for Nuclear Research, Dubna) for helpful discussions.

\section{REFERENCES}

[1] Zheng, L.X., O'Connell, M.J., Doorn, S.K., Liao, X.Z., Zhao, Y.H., Akhadov, E.A., Hoffbauer, M.A., Roop, B.J., et al. (2003) Nature Materials, 3(10), 673-676.

[2] Mintmire, J.W., Dunlap, B.I. and White, C.T. (1992) Physical Review Letters, 68(5), 631-634.

[3] Dekker, C. (1999) Carbon nanotubes as molecular quantum wires. Physics Today, 52(5), 22-28. http://www.physicstoday.org/vol-56/iss-2/pdf/Vol52no5p22-28.pdf

[4] Martel, R., Derycke, V., Lavoie, C., Appenzeller, J., Chan, K.K., Tersoff, J. and Avouris, Ph. (2001) Ambipolar electrical transport in semiconducting single-wall carbon nanotubes. Physical Review Letters, 87(25), 256805.

[5] Tersoff, J. (1989) Modeling solid-state chemistry: Interatomic potentials for multicomponent systems. Physical Review B, 39(8), 5566-5568.

[6] Affholter, K.A., Henderson, S.J., Wignall, G.D., Bunick, G.J., Haufler, R.E. and Compton, R.N. (1993) Structural characterization of $\mathrm{C}_{60}$ and $\mathrm{C}_{70}$ fullerenes by small-angle neutron scattering. Journal of Chemical Physics, 99(11), 9224-9229.

[7] Melnichenko, Y.B., Wignall, G.D., Compton, R.N. and Bakale, G. (1999) Characterization of fullerenes and fullerene derivatives by small-angle neutron scattering and transmission measurements. Journal of Chemical Physics, 111(10), 4724.

[8] Smorenburg, H.E., Crevecoeur, R.M., de Schepper, I.M. and de Graaf, L.A. (1995) Structure and dynamics of $\mathrm{C}_{60}$ molecules in liquid $\mathrm{CS}_{2}$ from neutron scattering. Physical Review E, 52(3), 2742-2752.

[9] Migliardo, F., Magazu, V. and Migliardo, M. (2004) Structural properties of $\mathrm{C}_{60}$ in solution. Journal of Molecular Liquids, 110(1-3), 3-6.

[10] Tomiyami, T., Uchiyama, S. and Shinohara, H. (1997) Solubility and partial spartial volume of $\mathrm{C}_{60}$ and $\mathrm{C}_{70}$ Chemical Physics Letters, 264, 143-148.

[11] Bokare, A.D. and Patnaik, A. (2003) Microscopic diffusion model applied to $\mathrm{C}_{60}$ fullerene fractals in carbon disulphide solution. Journal of Chemical Physics, 119(8), 4529-4538.

[12] Teterev, Yu., Aksenov, V.L., Avdeev, M.V. and Kholmurodov, Kh.T. (2007) Organization of solvent at interface with fullerene in solution C60/carbon disulfide by molecular bynamics simulations. In: Kholmurodov, K., Ed., Molecular Simulation Studies in Materials and Biological Sciences, Nova Science Publishers, New York, 129-134.

[13] Smith, W. and Forester, T.R. (1996) The DL POLY molecular simulation package. Journal of Molecular Graphics, 14(3), 136-141.

[14] Smith, W., Forester, T.R. and Todorov, I.T. (2008) The DL poly 2 user manual. Version 2.19, STFC Daresbury Laboratory Daresbury, Warrington WA4 4AD Cheshire, UK.

[15] Kholmurodov, K., Smith, W., Yasuoka, K. and Ebisuzaki, T. (2000) Highly vectorised "link-cell" Fortran code for the DL POLY molecular dynamics simulation package. Computer Physics Communications, 125(1-3), 167-192. 